This item was submitted to Loughborough's Research Repository by the author.

Items in Figshare are protected by copyright, with all rights reserved, unless otherwise indicated.

\title{
Relationships between fast bowling technique and ball release speed in cricket
}

PLEASE CITE THE PUBLISHED VERSION

http://journals. humankinetics.com/jab-current-issue/jab-volume-29-issue-1-february/the-relationships-betweenfast-bowling-technique-and-ball-release-speed-in-cricket

\section{PUBLISHER}

(c) Human Kinetics, Inc.

\section{VERSION}

VoR (Version of Record)

\section{LICENCE}

CC BY-NC-ND 4.0

\section{REPOSITORY RECORD}

Worthington, Peter J., Mark A. King, and Craig A. Ranson. 2019. "Relationships Between Fast Bowling Technique and Ball Release Speed in Cricket”. figshare. https://hdl.handle.net/2134/12151. 
This item was submitted to Loughborough's Institutional Repository (https://dspace.lboro.ac.uk/) by the author and is made available under the following Creative Commons Licence conditions.

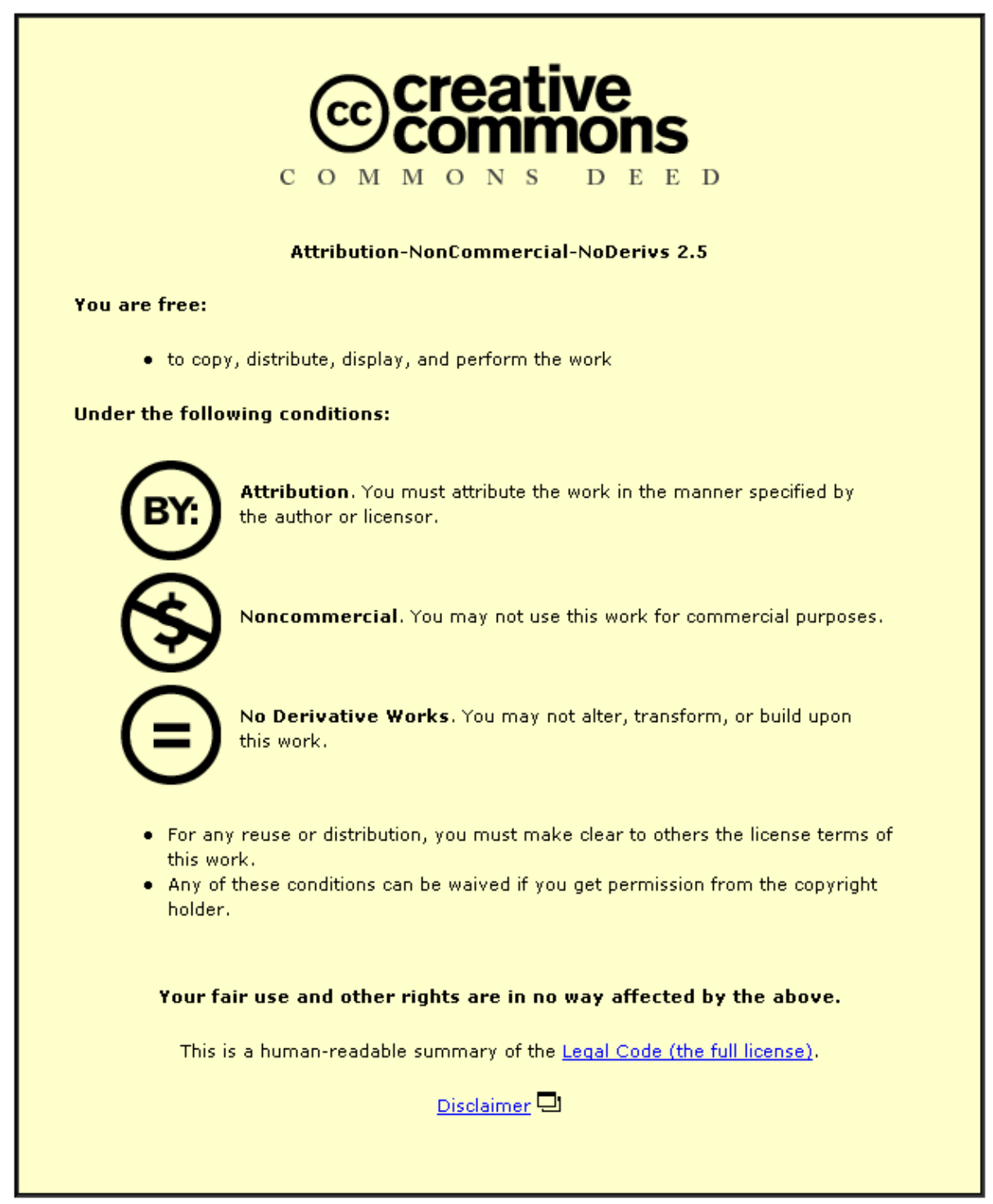

For the full text of this licence, please go to: http://creativecommons.org/licenses/by-nc-nd/2.5/ 


\title{
Relationships Between Fast Bowling Technique and Ball Release Speed in Cricket
}

\author{
Peter J. Worthington, ${ }^{1}$ Mark A. King, ${ }^{1}$ and Craig A. Ranson ${ }^{2}$ \\ ${ }^{1}$ Loughborough University; ${ }^{2}$ Cardiff Metropolitan University
}

\begin{abstract}
The aim of this study was to identify the key aspects of technique that characterize the fastest bowlers. Kinematic data were collected for 20 elite male fast bowlers with 11 kinematic parameters calculated, describing elements of fast bowling technique that have previously been linked to ball release speed. Four technique variables were identified as being the best predictors of ball release speed, explaining $74 \%$ of the observed variation in ball release speed. The results indicate that the fastest bowlers have a quicker run-up and maintain a straighter knee throughout the front foot contact phase. The fastest bowlers were also observed to exhibit larger amounts of upper trunk flexion up to ball release and to delay the onset of arm circumduction. This study identifies those technique variables that best explain the differences in release speeds among fast bowlers. These results are likely to be useful in both the coaching and talent identification of fast bowlers.
\end{abstract}

Keywords: cricket, fast bowling, ball speed, linear regression

Ball release speed is a major contributor to fast bowling success, reducing the time the batsman has to assess the path of the ball and make decisions regarding which shot to play. The fastest bowlers release the ball at speeds in excess of $40 \mathrm{~m} \cdot \mathrm{s}^{-1}(90 \mathrm{mph})$. Numerous previous investigations have sought to identify those aspects of fast bowling technique that are linked to ball release speed, in an attempt to understand how certain bowlers are able to release the ball at faster speeds than others. However, the interactions between the various aspects of technique that have been associated with higher ball speed have not been accounted for and so limiting their application to the coaching and performance of fast bowling.

A strong correlation has been reported between run-up speed (during the predelivery stride) and ball release speed. ${ }^{1,2}$ This suggested that run-up speed should be sufficient to produce as high a linear velocity of the body as possible, while enabling the correct delivery technique to be adopted. Other studies have investigated relationships between ball release speed and front knee motion during the front foot contact phase of the bowling action. Faster release speeds have been associated with a more extended front knee at the instant of ball release. ${ }^{3,4}$ Similarly, faster bowlers have been observed to have larger amounts of front knee extension during

Peter J. Worthington and Mark A. King (Corresponding Author) are with the School of Sport, Exercise and Health Sciences, Loughborough University, Loughborough, UK. Craig A. Ranson is with the Cardiff School of Sport, Cardiff Metropolitan University, Cardiff, Wales. the period from full foot contact (front foot flat, FFF) until ball release..$^{5}$ It has been suggested that a front knee that extends, or is already extended, during the front foot contact phase may permit a more efficient transfer of energy to the ball and facilitate faster ball release speeds. ${ }^{5-7}$ However, there is currently no firm agreement regarding the importance of the motion of the front knee in determining ball release speed.

Bowling action classification (front-on, midway or side-on shoulder girdle alignment at back foot contact, BFC) was not observed to be linked to ball release speed, ${ }^{8}$ nor was the amount of shoulder girdle counter-rotation. ${ }^{5}$ However, a correlation between ball release speed and the timing of the maximum pelvis-shoulder separation angle during the delivery stride was observed. ${ }^{5}$ Those bowlers whose maximum separation angle occurred later in the delivery action (relative to the instant of front foot contact, FFC) bowled faster. Similarly the position of the arm at FFC was a good predictor of release speed, with faster bowlers delaying the onset of arm circumduction. ${ }^{9}$ However, no subsequent research has been identified as supporting this. ${ }^{10}$

A link between increased shoulder girdle and upper trunk rotation (shoulder girdle forward rotation) preceding ball release (BR) and faster release speed has been reported. ${ }^{5}$ Increased flexion of the trunk (between FFC and BR) has also been reported to provide a significant contribution to ball release speed, contributing an estimated $11-13 \%$ of the final release speed. ${ }^{6,11}$ A number of studies have identified links between the position of the arm (and ball) at the instant of ball release and the release speed. Faster bowlers release the ball with the arm further out in front of the line of the trunk. ${ }^{6,11-14}$ 
Although previous investigations have addressed the question of which aspects of fast bowling technique determine the speed at which bowlers release the ball, there remains little agreement between studies. Almost all have used linear correlations to assess whether a particular aspect of bowling technique was independently linked to ball release speed, with the more recent studies introducing increasing numbers of aspects of bowling technique into the analysis. It is hypothesized that variation in bowling speed can be explained using a small number of kinematic variables. The aim of the current study was to identify the key kinematic parameters of a fast bowler's technique that can predict bowling speed.

\section{Methods}

Twenty elite male fast bowlers (mean \pm standard deviation: age $20.1 \pm 2.6$ years; height $1.88 \pm 0.08 \mathrm{~m}$; body mass $81.5 \pm 7.1 \mathrm{~kg}$ ) participated in this investigation. All bowlers were members of the England and Wales Cricket Board's (ECB) elite fast bowling group-being either current England bowlers or identified as likely to play for England in the next three to five years. All bowlers were deemed fit to bowl by their County or National Team Physiotherapist. The testing procedures were explained to each bowler and informed consent was obtained in accordance with the guidelines of the Loughborough University Ethical Advisory Committee. All bowlers conducted a thorough warm-up before data collection. Each bowler performed six maximum velocity deliveries of good length; these were recorded using an 18 camera $\left(\mathrm{M}^{2} \mathrm{MCam}\right)$ Vicon Motion Analysis System (OMG Plc, Oxford, UK) operating at $300 \mathrm{~Hz}$. Data were collected in an indoor cricket facility (Figure 1); bowlers were able to use a full length run-up on a standard sized artificial cricket pitch.

Forty-seven $14 \mathrm{~mm}$ retro-reflective markers were attached to each bowler (Figure 2), positioned over bony

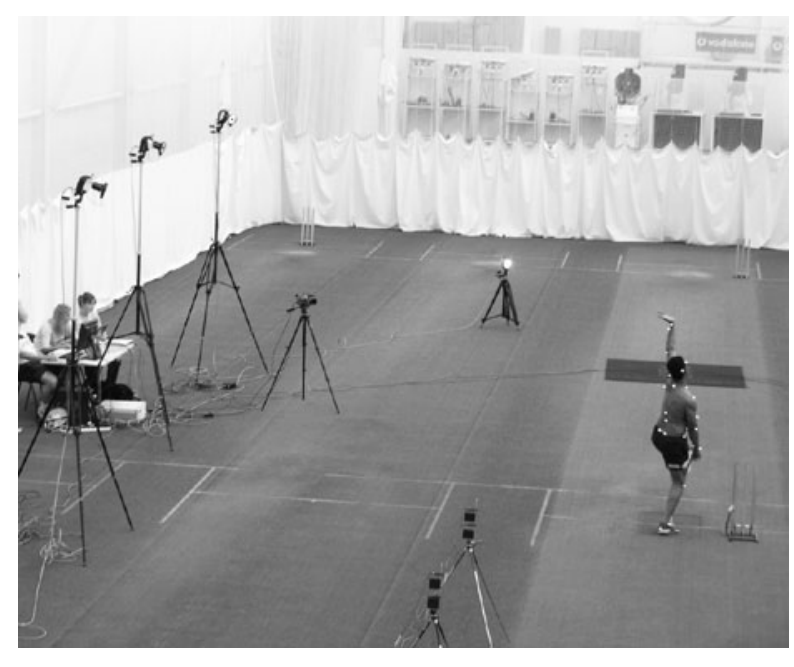

Figure 1 - The data collection environment.

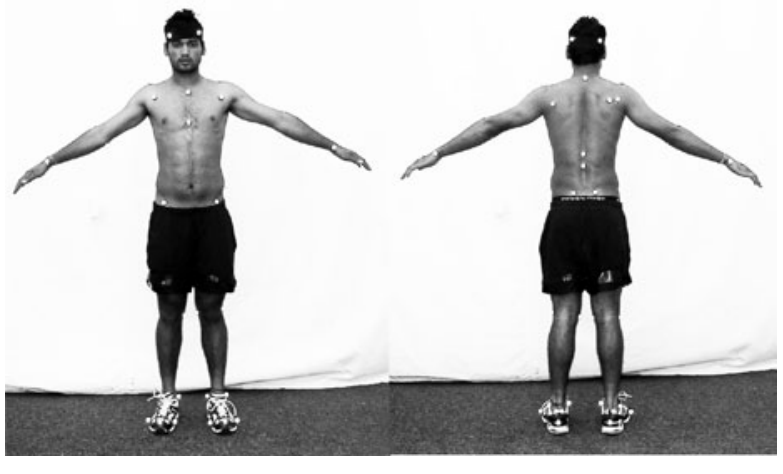

Figure 2 - The full body marker set used.

landmarks. An additional marker, a $15 \times 15 \mathrm{~mm}$ patch of $3 \mathrm{M}$ Scotch-Lite reflective tape, was attached to the ball to enable release speed and the instant of release to be determined. The ankle, knee, shoulder, elbow and wrist joint centers were calculated from a pair of markers placed across the joint, positioned such that their midpoint coincided with the joint center. ${ }^{15}$ The hip joint centers were calculated from markers placed over the left and right anterior superior iliac spine and the left and right posterior superior iliac spine. ${ }^{16}$ Lower and upper trunk motions were defined using the four markers on the pelvis in addition to markers placed over the cephalad and caudad ends of the sternum as well as the spinous processes of $\mathrm{L} 1, \mathrm{~T} 10$ and $\mathrm{C} 7 . .^{15}$

Static and range of motion (ROM) trials were performed for each bowler, allowing body segment lengths and a neutral spine position to be calculated. ${ }^{17}$ Anthropometric measurements were taken, ${ }^{18}$ enabling subjectspecific segmental inertia parameters to be determined for each bowler.

Three bowling trials for each bowler (maximum velocity deliveries with good Vicon data) were manually labeled and processed within Vicon's software (OMG Plc, Oxford, UK). The instants of back foot contact (BFC), front foot contact (FFC) and front foot flat (FFF) were identified using the motions of the markers on the foot. Ground contact was defined as the first frame in which the foot's motion was visually observed to change due to contact with the ground. FFF corresponded to the first frame in which the forefoot was on the ground. Ball release was identified using the time-history of the distance between the ball marker and the midpoint of a pair of markers placed over the wrist. The frame corresponding to ball release was defined as the first frame in which this distance increased by more than $20 \mathrm{~mm}$ relative to the distance in the previous image. All marker trajectories were filtered using a recursive fourth-order low-pass Butterworth filter with a cut-off frequency of $30 \mathrm{~Hz} .{ }^{19}$

Local reference frames were defined comprising a three-dimensional full-body 18 segment representation of a bowler (head and neck; upper trunk; lower trunk; pelvis; $2 \times$ arm; $2 \times$ lower-arm; $2 \times$ hand; $2 \times$ upper-leg; 
$2 \times$ lower-leg; and $2 \times$ two-segment foot). These reference frames were defined using three markers on the segment itself, allowing segment orientations and joint angles to be calculated. The z-axis pointed upwards along the longitudinal axis of the segment, the $\mathrm{x}$-axis pointed toward the subject's right (flexion-extension axis of the joint) and the y-axis pointed forward. Similarly, a global coordinate system was defined with the y-axis pointing down the wicket (toward the batsman), the $\mathrm{x}$-axis pointing to the right and the z-axis representing the upwards vertical. Joint angles were calculated as Cardan angles, defining the rotation applied to the parent coordinate system (proximal segment) to bring it into coincidence with the coordinate system of the child segment (distal segment). Rotation angles were calculated using an xyz sequence-corresponding to flexion-extension, abduction-adduction, and longitudinal rotation, respectively.

Eleven kinematic parameters were calculated for each trial, describing elements of fast bowling technique that have been linked to ball release speed in previous studies: run-up speed (horizontal); knee angle at FFC and BR; knee flexion and extension (FFF till BR); shoulder girdle forward rotation; upper trunk flexion (FFC till BR); shoulder angle at FFC and BR; minimum pelvis-shoulder separation angle and its timing relative to FFC.

The horizontal run-up speed (in the global y-direction) was calculated as the mean mass center velocity over a period of 18 frames $(0.060 \mathrm{~s})$ immediately before the instant of BFC. Similarly, ball release speed was calculated using the motion of the ball marker during a period of 10 frames $(0.033 \mathrm{~s})$ from the instant of BR along with constant acceleration equations for the vertical velocity of the ball at BR. Angles describing the front knee (straight $=180^{\circ}$, flexed $<180^{\circ}$ ), upper trunk (straight $=0^{\circ}$ ) and shoulder (anatomical position $=0^{\circ}$, Figure 3 ) corresponded to the anatomical flexion / extension angle of the joint. The orientation angle of the upper trunk was normalized using a neutral position of the spine. ${ }^{17}$ An upright position of the upper trunk was defined to correspond to an angle of $0^{\circ}, 0^{\circ}, 0^{\circ}$ about the $\mathrm{x}, \mathrm{y}$ and $\mathrm{z}$-axes of the lower trunk segment.

The alignment of the shoulders and pelvis were calculated by projecting their respective joint centers onto a horizontal plane (Figure 4). A bowler facing directly down the wicket (front-on) was defined to have a shoulder and pelvis projection angle of $270^{\circ}$, standing in a purely side-on position corresponded to a projection angle of $180^{\circ} .5$ Shoulder girdle forward-rotation was defined as the change in shoulder projection angle from the most side-on position to the orientation at BR. The pelvisshoulder separation angle was calculated by subtracting the pelvis projection angle from the shoulder projection angle. The minimum pelvis-shoulder separation angle was calculated for each trial, in addition to the timing of this instant relative to FFC.

All statistical analyses were performed within SPSS v.17 (SPSS Corporation, USA). The variation observed in each technique parameter (including ball speed) was assessed using analysis of variance (ANOVA). The between-trial variability was compared with the betweenbowler variability and was found to be much smaller. In
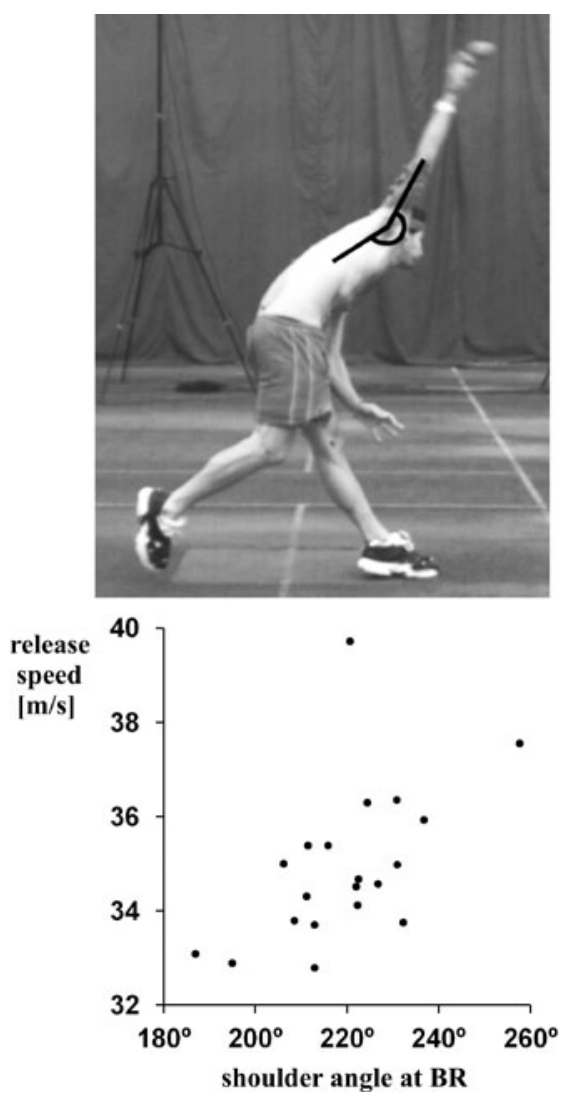

Figure 3 - Illustration of the shoulder angle $\left(195^{\circ}\right)$ calculated at ball release and ball release speed plotted against shoulder angle.

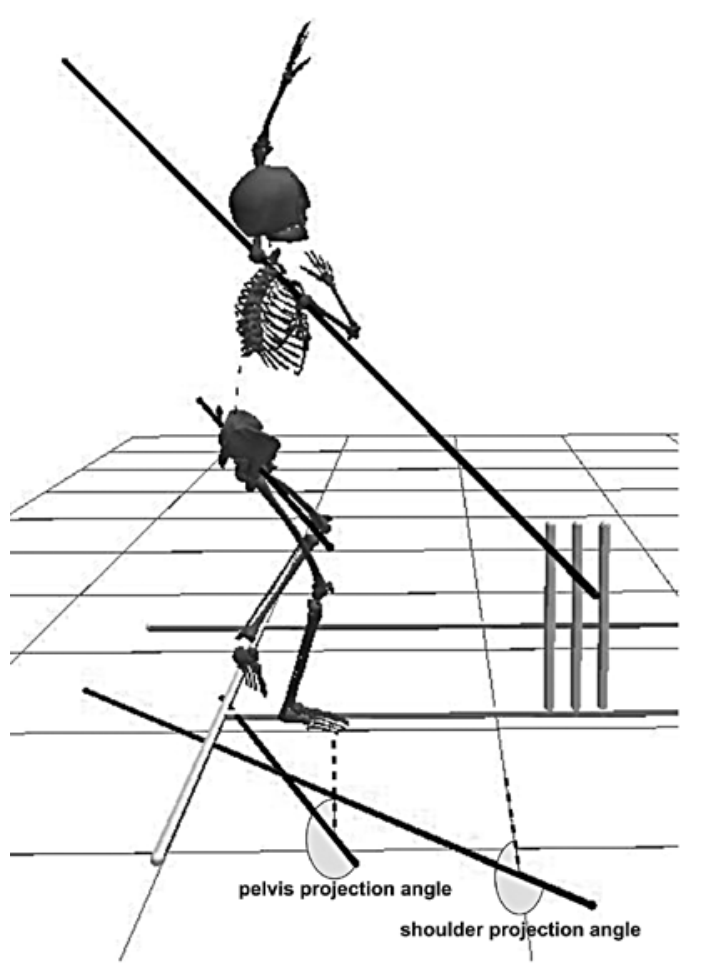

Figure 4 - The shoulder and pelvis projection angles for a bowler at back foot contact. 
particular, the between-trial standard deviation of the observations ranged from 8.3 to $22.5 \%$ (mean $13.3 \%$ ) of the between-bowler variation for the parameters used in this study. This corresponded to an intra-class correlation coefficient (ICC) of 0.95-0.99 (mean 0.98) and indicates good between-trial repeatability for all technique parameters calculated. ${ }^{20}$ As a consequence the three trials analyzed were averaged for each parameter to provide representative data for each bowler. The effect of technique variables on ball release speed were addressed using forward stepwise linear regression. The requirement for the inclusion of a variable in the regression equation was $P<.10$.

\section{Results}

The twenty bowlers participating in this study had ball release speeds of $32.8-39.7 \mathrm{~m} \cdot \mathrm{s}^{-1}$ (mean $34.9 \pm 1.7$ $\left.\mathrm{m} \cdot \mathrm{s}^{-1}\right)$. Six bowlers had a front-on shoulder alignment at $\mathrm{BFC}$ and the remaining fourteen were midway. Using the front knee technique classification system, ${ }^{5}$ the bowlers were classified as: nine flexor-extenders; six flexors; three extenders; and two using a constant brace. The bowlers had run-up speeds in the range $4.8-6.8 \mathrm{~m} \cdot \mathrm{s}^{-1}$ (Table 1).

The best individual predictor of ball release speed was the shoulder angle at ball release (BR), explaining $30 \%$ of the variation in release speed (Table 2, Figure 5a). An arm that was further back at BR (ie, a larger shoulder angle, Figure 3), relative to the line of the upper trunk characterized the fastest bowlers. Adding run-up speed into the regression equation increased the percentage variation explained to $57 \%$ (Figure 5b), with the fastest bowlers having a quicker run-up. The regression equation was further improved by including the front knee angle at BR (66\% of the variation explained, Figure 5c), with a more extended front knee being associated with faster release speeds. There was insufficient evidence $(P$ $>$.10) supporting the addition of a fourth variable into the regression equation using forward stepwise regression (Table 2). However, a four-parameter regression equation with significant $P$-values for each variable was found by trying all combinations of the eleven kinematic

Table 1 Range, mean and standard deviation of the 11 technique parameters

\begin{tabular}{lcc}
\hline Technique Variable & Range & Mean \pm SD \\
\hline Run-Up speed $\left(\mathrm{m} \cdot \mathrm{s}^{-1}\right)$ & $4.77-6.76$ & $5.79 \pm 0.58$ \\
Knee angle at FFC $\left(^{\circ}\right)$ & $148.3-172.7$ & $164.1 \pm 6.1$ \\
Knee angle at BR $\left(^{\circ}\right)$ & $120.3-186.2$ & $167.3 \pm 18.8$ \\
Knee flexion from FFF till BR $\left(^{\circ}\right)$ & $0.0-44.8$ & $17.5 \pm 11.2$ \\
Knee extension from FFF till BR $\left(^{\circ}\right)$ & $0.3-26.3$ & $11.9 \pm 7.4$ \\
Shoulder girdle forward-rotation $\left(^{\circ}\right)$ & $80.6-143.4$ & $115.5 \pm 18.2$ \\
Upper trunk flexion from FFC till BR $\left(^{\circ}\right)$ & $11.2-50.6$ & $31.0 \pm 8.3$ \\
Shoulder angle at FFC $\left({ }^{\circ}\right)$ & $288.0-365.0$ & $331.2 \pm 22.1$ \\
Shoulder angle at BR $\left({ }^{\circ}\right)$ & $186.9-257.6$ & $219.4 \pm 15.3$ \\
Min. pelvis-shoulder separation $\left(^{\circ}\right)$ & -63.3 to -27.5 & $-39.6 \pm 9.6$ \\
Time of min. pelvis-shoulder separation $(\mathrm{s})$ & -0.020 to 0.057 & $0.031 \pm 0.019$ \\
\hline
\end{tabular}

Abbreviations: front foot contact (FFC); front foot flat (FFF); ball release (BR).

Table 2 Regression equations for release speed using stepwise linear regression

\begin{tabular}{clccc}
\hline $\begin{array}{c}\text { Number of } \\
\text { Parameters }\end{array}$ & Technique Parameter(s) & Coefficient & P-Value & $\begin{array}{c}\text { Percentage } \\
\text { Explained }\end{array}$ \\
\hline 1 & Shoulder angle at BR & 0.060 & .012 & 30.3 \\
2 & Shoulder angle at BR & 0.061 & .002 & 56.9 \\
& Run-Up speed & 1.485 & .005 & \\
3 & Shoulder angle at BR & 0.038 & .074 & \\
& Run-Up speed & 1.623 & .002 & 65.5 \\
& Knee angle at BR & 0.033 & .063 & \\
4 & Shoulder angle at BR & 0.035 & .084 & \\
& Run-Up speed & 1.555 & .002 & 70.1 \\
& Knee angle at BR & 0.029 & .087 & \\
& Shoulder angle at FFC & 0.017 & .150 & \\
\hline
\end{tabular}

Abbreviations: front foot contact (FFC); ball release (BR). 
parameters (four-parameter solution which explains the most variance in ball speed), this explained $74 \%$ of the variation in release speed (Table 3, Figure 5e). This consisted of: run-up speed; knee angle at BR; upper trunk flexion from FFC until BR; and shoulder angle at FFC. The fastest bowlers had more upper trunk flexion between FFC and BR and appeared to delay the onset of arm circumduction, indicated by a larger shoulder angle at FFC.

\section{Discussion}

Previous studies have identified various aspects of technique that are individually correlated with ball release speed. There is currently little consensus between studies and their findings have not been widely implemented in the coaching of fast bowlers as interactions between technique variables have not been accounted for. The current study addressed this issue using linear regression and those technique variables that best explain the differences in release speed among elite bowlers were identified. The results of this investigation suggest $74 \%$ of the variation in ball speed observed between bowlers can be explained using just four technique parameters. These parameters were: run-up speed; knee angle at ball release; upper trunk flexion from front foot contact until ball release; and shoulder angle at front foot contact.

The best individual predictor of release speed was the shoulder angle at BR. The fastest bowlers had their arm further back relative to their upper trunk as they released the ball (Figure 3). Although it might be expected that having the arm further forward would contribute more to ball speed, the fact that the arm is further back indicates the position the fastest bowlers adopt at BR as a consequence of their technique during the delivery stride. Previous studies have reported a trend for quicker bowlers to release the ball with the arm "further out in front of the line of the trunk."6,11-14 The results of the current study appear to contradict these observations which could be attributable to the low frequency kinematic data $(50 \mathrm{~Hz})$ used in previous studies or difficulty in identifying the instant of BR. However, it is more likely that previous researchers were referring to the orientation of the arm in relation to the vertical. The trend observed in the current study indicates that bowlers use upper trunk flexion to generate ball speed. Had the bowlers generated bowling arm speed using predominantly their shoulder muscles, it is unlikely their arm would be behind the line of the upper trunk at BR.

In the best four-parameter regression equation, the shoulder angle at BR was replaced by two parameters: the shoulder angle at FFC; and the amount of upper trunk flexion between FFC and BR. The inclusion of upper trunk flexion in the regression equation supports the suggestion that bowlers generate ball speed using upper trunk flexion. The mechanism by which the fastest bowlers have increased amounts of upper trunk flexion between FFC and BR is unclear and warrants further investigation. It is speculated that the increased levels of upper trunk flexion are a consequence of the "overall bowling action used" rather than due to increased muscular work in the torso. The inclusion of the shoulder angle at FFC in the fourparameter regression equation provides evidence that delaying the onset of arm circumduction (larger shoulder angle at FFC) enables bowlers to release the ball at faster speeds. This trend for the fastest bowlers to delay the motion of their bowling arm was previously reported by, ${ }^{9}$ but has not been reported in any other subsequent studies. A larger shoulder angle at FFC gives a larger range of motion at the shoulder over which to generate ball speed and may also allow greater torques to be exerted about the shoulder. The reason why the faster bowlers have a greater shoulder angle at FFC is not obvious and should be the topic of further study.

A more extended front knee at BR characterized the fastest bowlers. By planting their front leg at FFC, bowlers convert the linear momentum of their run-up into angular momentum about the front foot. Bowlers use the front leg to rapidly slow the linear velocity of the pelvis, which in turn drives the trunk forward about the pelvis. By maintaining a straight front knee throughout the front foot contact phase, bowlers are able to perform this more efficiently. ${ }^{7}$ These bowlers can be identified as those who have the most extended front knee at the instant of BR.

Run-up speed was positively correlated with ball release speed, as reported previously. ${ }^{1,2}$ Bowlers with a quicker run-up have a greater amount of linear momentum that can potentially be converted into ball speed. There is likely to be an optimum run-up speed, beyond which ball release speed decreases, ${ }^{21}$ as bowlers are unable to maintain the technique required to control the additional run-up speed.

Table 3 The best four-parameter regression equation for release speed

\begin{tabular}{lccc}
\hline Technique Parameter(s) & Coefficient & $\boldsymbol{P}$-Value & $\begin{array}{c}\text { Percentage } \\
\text { Explained }\end{array}$ \\
\hline Run-Up speed & 1.529 & .001 & \\
Knee angle at BR & 0.042 & .004 & 73.6 \\
Upper trunk flexion (FFC - BR) & 0.070 & .029 & \\
Shoulder angle at FFC & 0.028 & .023 & \\
\hline
\end{tabular}

Abbreviations: front foot contact (FFC); ball release (BR). 

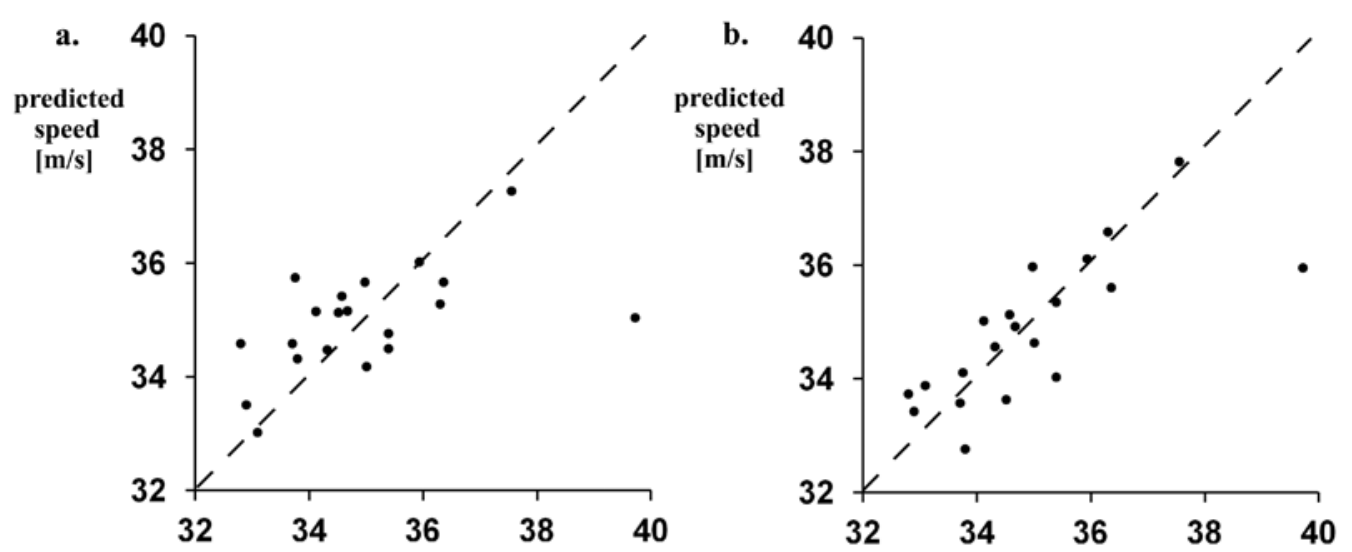

$30.3 \%$ of the variation in release speed explained by: shoulder angle at $\mathrm{BR}$

$56.9 \%$ of the variation in release speed explained by: shoulder angle at $\mathrm{BR}$ run-up speed
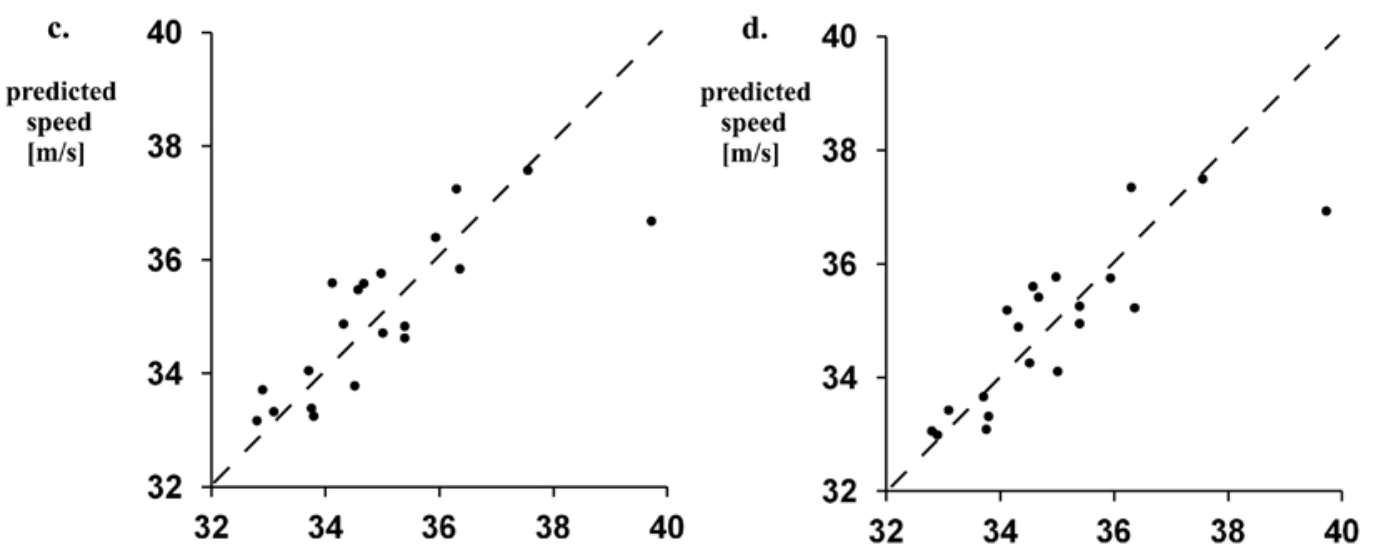

$65.5 \%$ of the variation in release speed explained by: shoulder angle at BR run-up speed

knee angle at $\mathrm{BR}$

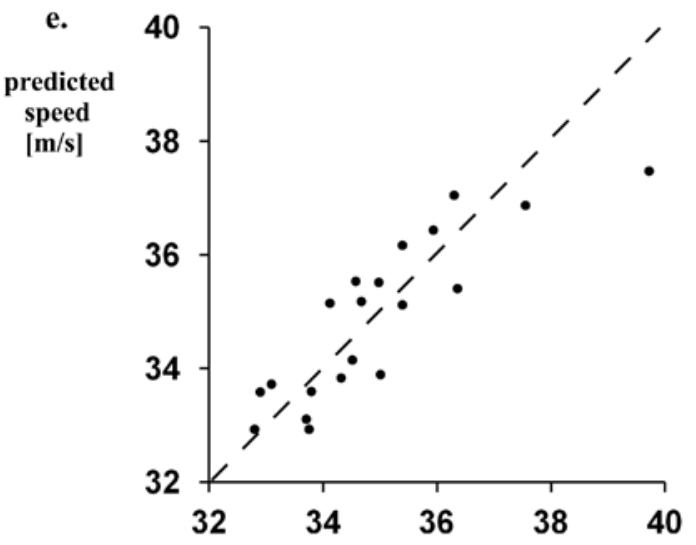

$70.1 \%$ of the variation in release speed explained by: shoulder angle at $B R$ run-up speed knee angle at $B R$ shoulder angle at FFC

$73.6 \%$ of the variation in release speed explained by:

run-up speed

knee angle at $B R$

upper trunk flexion (FFC - BR)

shoulder angle at FFC

Figure 5 - Predicted ball speed against actual release speed for the four stepwise solutions (a-d; Table 2); and the best overall four-parameter solution (e; Table 3). With a higher percentage of the variation in release speed explained the closer the data points lie to the dashed line $\mathrm{y}=\mathrm{x}$ (predict speed $=$ actual speed). 
Small sample sizes are a common problem when studying elite populations; the sample of 20 elite fast bowlers used in the current study represents a relatively large sample for this particular population. Although the sample size limits the power of the statistical tests that can be conducted, there is sufficient data to enable regression to be used to identify those variables that best explain ball speed. This represents an important tool in this type of situation, enabling the most important technique variables to be identified from those found to be correlated to ball speed in previous studies. It is important with multiple regression not to attribute too much meaning to the order of entry of variables, their marginal contributions to the $R^{2}$, or to their relative significance levels. In this paper multiple regression analysis has been used appropriately, alongside more traditional biomechanical research evidence, and this has led to insights that would not be achieved using simple correlations. In particular, the results provide a relationship between fast bowling technique and release speed and are likely to provide a valuable framework on which the coaching of fast bowling can be based. The significance levels required for inclusion of technique variables in the regression equation were relaxed to $10 \%$ to increase the ability of the tests to detect relationships of potential interest.

The $74 \%$ of variation in release speed explained by the four-parameter regression equation suggests the key aspects of technique have been identified for a group of elite fast bowlers. In particular, the fastest bowlers have a quicker run-up, maintain a straighter knee throughout the front foot contact phase, have larger amounts of upper trunk flexion up to ball release and appear to delay the onset of arm circumduction. The results of this investigation are likely to be very useful in the coaching and conditioning of fast bowlers in addition to guiding talent identification among young bowlers. Future studies should address whether these results are independent of a bowler's physical characteristics, for example whether taller bowlers generate ball speed differently to bowlers who are relatively shorter.

\section{Acknowledgment}

This project was funded by the England and Wales Cricket Board (ECB).

\section{References}

1. Glazier PS, Paradisis GP, Cooper SM. Anthropometric and kinematic influences on release speed in men's fast-medium bowling. J Sports Sci. 2000;18:1013-1021. PubMed doi:10.1080/026404100446810

2. Elliott BC, Foster DH. Fast bowling technique. In: Elliott B, Foster D, Blanksby B, eds. Send the Stumps Flying: The Science of Fast Bowling. Nedlands, WA: University of Western Australia Press; 1989:26-36.

3. Burden AM, Bartlett RM. A kinematic investigation of elite fast and fast medium cricket bowlers. In Nosek M, Sojka D, Morrison WE, Susanka P, eds. Proceedings of the VIIIth International Symposium of the Society of Biomechanics in Sports. Conex: Prague; 1990:41-46.
4. Wormgoor S, Harden L, Mckinon W. Anthropometric, biomechanical, and isokinetic strength predictors of ball release speed in high-performance cricket fast bowlers. J Sports Sci. 2010;28:957-965. PubMed doi:10.1080/02640411003774537

5. Portus MR, Mason BR, Elliott BC, Pfitzner MC, Done RP. Technique factors related to ball release speed and trunk injuries in high performance cricket fast bowlers. Sports Biomech. 2004;3:263-283. PubMed doi:10.1080/14763140408522845

6. Elliott BC, Foster D, Gray S. Biomechanics and physical factors affecting fast bowling. Aust J Sci Med Sport. 1986;18:16-21.

7. Ferdinands R, Marshall RN, Kersting U. Centre of mass kinematics of fast bowling in cricket. Sports Biomech. 2010;9:139-152. PubMed doi:10.1080/14763141.2010. 523844

8. Stockill NP, Bartlett RM. Progress report on the National Cricket Association Sports Science Support Programme fast bowling project. Cricket Coach. 1992;22:1165-1172.

9. Tyson F. Complete Cricket Coaching. Melbourne: Thomas Nelson Ltd; 1976.

10. Bartlett RM, Stockill NP, Elliott BC, Burnett AF. The biomechanics of fast bowling in men's cricket: A review. J Sports Sci. 1996;14:403-424. PubMed doi:10.1080/02640419608727727

11. Davis K, Blanksby B. A cinematographical analysis of fast bowling in cricket. Aust J Health Phy Educ Rec. 1976;71:9-15.

12. Burden AM, Bartlett RM. A kinematic analysis of fast bowling in cricket. J Sports Sci. 1989;7:75.

13. Foster D, John D, Elliott B, Ackland T, Fitch K. Back injuries to fast bowlers in cricket: A prospective study. $\mathrm{Br}$ J Sports Med. 1989;23:150-154. PubMed doi:10.1136/ bjsm.23.3.150

14. Burden AM. An electromyographical and cinematographical analysis of fast-medium bowling in cricket. Unpublished Master's thesis, University of Salford; 1990.

15. Ranson C, King M, Burnett A, Worthington P, Shine K. The effect of coaching intervention on elite fast bowling technique over a two year period. Sports Biomech. 2009;8:261274. PubMed doi:10.1080/14763140903469908

16. Davis RB, Ounpuu S, Tyburski D, Gage JR. A gait analysis data collection and reduction technique. Hum Mov Sci. 1991;10:575-587. doi:10.1016/0167-9457(91)90046-Z

17. Ranson CA, Burnett AF, King M, Patel N, O'Sullivan PB. The relationship between bowling action classification and three-dimensional lower trunk motion in fast bowlers in cricket. J Sports Sci. 2008;26:267-276. PubMed doi:10.1080/02640410701501671

18. Yeadon MR. The simulation of aerial movementII: A mathematical inertia model of the human body. J Biomech. 1990;23:67-74. PubMed doi:10.1016/0021-9290(90)90370-I

19. Winter DA. Biomechanics and Motor Control of Human Movement. New York: Wiley; 1990.

20. Norton K, Marfell-Jones M, Whittingham N, et al. Anthropometric assessment protocols. In: Gore C, ed. Physiological Tests for Elite Athletes. 1st ed. Champaign, IL: Human Kinetics; 2000:66-85.

21. Brees AJ. A cinematographical analysis of the effect of varying the run-up speed on ball release velocity, accuracy and body kinematics of fast bowling in cricket. Unpublished undergraduate dissertation, Crewe and Alsager College of Higher Education; 1989. 